\title{
Assessment of menopausal symptoms using modified Menopause Rating Scale (MRS) among middle age women in Kuching, Sarawak, Malaysia
}

\author{
Syed Alwi Syed Abdul Rahman ${ }^{1 *}$, Siti Rubiah Zainudin², Verna Lee Kar Mun³
}

\begin{abstract}
Background: Menopausal symptoms can be assessed by several tools, and can be influenced by various sociodemographic factors.

Objectives: To determine the commonly reported menopausal symptoms among Sarawakian women using a modified Menopause Rating Scale (MRS).

Methods: By using modified MRS questionnaire, 356 Sarawakian women aged 40-65 years were interview to document of 11 symptoms (divided into somatic, psychological and urogenital domain) commonly associated with menopause.

Results: The mean age of menopause was 51.3 years (range 47 - 56 years). The most prevalent symptoms reported were joint and muscular discomfort (80.1\%); physical and mental exhaustion (67.1\%); and sleeping problems (52.2\%). Followed by symptoms of hot flushes and sweating (41.6\%); irritability (37.9\%); dryness of vagina (37.9\%); anxiety (36.5\%); depressive mood (32.6\%). Other complaints noted were sexual problem (30.9\%); bladder problem (13.8\%) and heart discomfort (18.3\%). Perimenopausal women $(n=141)$ experienced higher prevalence of somatic and psychological symptoms compared to premenopausal $(n=82)$ and postmenopausal $(n=133)$ women. However urogenital symptoms mostly occur in the postmenopausal group of women.

Conclusions: The prevalence of menopausal symptoms using modified MRS in this study correspond to other studies on Asian women however the prevalence of classical menopausal symptoms of hot flushes, sweating was lower compared to studies on Caucasian women.
\end{abstract}

\section{Background}

Menopause which is defined as complete cessation of menstruation for twelve months or more is a normal physiological change experienced by middle age women. Some of menopausal symptoms experienced by these women can be severe enough to affect their normal daily activities. Unfortunately majority of these women are not aware of the changes brought about by menopause [1-4]. These symptoms are directly resulted from depletion of estrogen level as women approaches menopausal stage and some of these women begin to experiences these menopausal symptoms early in the perimenopausal phase. The common climacteric

\footnotetext{
* Correspondence: sarsalwi@fmhs.unimas.my

'Department of Family Medicine, Faculty of Medicine and Health Sciences Universiti Malaysia Sarawak, Kuching, Malaysia
}

symptoms experienced by them can be group into: vasomotor, physical, psychological or sexual complaints. It was also noted in some postmenopausal women with long term estrogen deficiency, changes to the cardiovascular or bone which leads to osteoporosis has been established. It is well documented that menopausal symptoms experienced by women affect their quality of life [5-7]. Many published reports shows variations in menopausal symptoms between Asian and Caucasians women, Asian women suffer lesser of somatic and psychological symptoms when compared to their western counterparts [8-11].

Studies shown that perimenopausal and postmenopausal women have more menopausal complaints compared to premenopausal women. They were noted to complain significantly more of vasomotor, sexual and 
psychological symptoms compared to premenopausal women [12-14].

Various tools or instruments have been designed to measure and assess symptoms during the menopausal transition, among them is Menopause Rating Scale (MRS) which is designed to assess menopause specific health related quantity of life (QoL) to measure the severity of age/menopause-related complaints by rating a profile of symptoms $[15,16]$.

Limited research data were available regarding menopausal symptoms experienced by women in Malaysia. Although menopause related symptoms have been extensively studied in the western countries, not much data were available in Asia especially in South East Asia including Malaysia. All previous studies on menopausal in Malaysia were conducted in Peninsular Malaysia [17-21].

This study was conducted in Kuching, which is the capital city of Sarawak, the largest state in Malaysia and situated in the Island of Borneo.

There is no documented or published data on menopausal study in Sarawak, and this study aimed to document the menopausal-related symptoms among middle age women of Kuching, Sarawak by using modified Menopause Rating Scale.

\section{Methods}

\section{Subjects and Setting}

This is a cross-sectional study. Conducted from the month of January 2007 to November 2007. This study was approved by Universiti Kebangsaaan Malaysia Research and Ethical Committee. The interview was carried out among women ages of 40 to 65 years who visited the government health centers around Kuching, Sarawak, Malaysia. These health centers which provided primary care services are government healthcare centers under the Ministry of Health, Malaysia. The inclusion criteria consisted of women between the ages of 40 to 65 years who had given consent to participate in this study. Pregnant and breast feeding women, women with uncontrolled medical conditions such as hypertension, diabetes mellitus or heart disease, or who were undergoing treatment for cancer, or were in remission, or who had history of drug or alcohol abuse and on hormone replacement therapy were excluded from the study.

\section{Instrument and data collection}

Questionnaires were divided into three sections:

(1) Socio-demographic data of the women, which included: age, race, religion, marital status, educational level, occupation and average household income.

(2) Menopausal status of the women: The menopausal status was classified according to STRAW (Stages of
Reproductive Aging Workshop) classification which divided menopause staging into: Postmenopausal; no menstrual bleeding in the previous/last 12 months. Late perimenopause; had menstruation in the previous/last 212 months but not in the previous/last 2 months. Early perimenopause; had increasing irregularity of menses without skipping periods ( 7 days difference from the beginning of a given cycle to the next, experienced after the previously regular cycle and Premenopause; minor changes in cycle length particularly decreasing length of the cycle. To aid statistical analysis, the early and late perimenopausal transition stages were grouped together as perimenopausal stage [22,23].

(3) Menopause Rating Scale (MRS) questionnaire were used as a basis for assessing menopausal symptoms in this study, this is a self-administered instrument which has been widely used and validated and have been used in many clinical and epidemiological studies, and in research on the etiology of menopausal symptoms to assess the severity of menopausal symptoms [16].

The MRS is composed of 11 items and was divided into three subscales:

(a) somatic-hot flushes, heart discomfort/palpitation, sleeping problems and muscle and joint problems; (b) psychological-depressive mood, irritability, anxiety and physical and mental exhaustion and (c) urogenital-sexual problems, bladder problems and dryness of the vagina. Each of the eleven symptoms contained a scoring scale from "0" (no complaints) to "4" (very severe symptoms). The questionnaire was first translated into Malay language by a group of experienced health-workers and language experts, and then translated back to English to validate whether the original meaning of the questionnaire was maintained in the translation; a pilot study was done on 60 women to validate the translated MRS questionnaires. The women were asked whether or not they had experienced the 11 menopausal symptoms shown in the MRS in the previous one month (30 days), however it was noted from the pilot study, these women had difficulties in rating the scale themselves, in order to minimize these difficulties, a face-to-face interviewed was done rather than using self-administered respond. Modifications were also made on the grading method of each item due to difficulties faced by the participant in identifying the severity of the symptoms hence the grading was modified to "present" or "absent" of symptoms. Reliability analysis was performed on the modified Menopause Rating Scale questionnaires with Cronbach's alpha of somatic subscale 0.712 , psychological subscale 0.743 and urogenital subscale 0.821 . Therefore, this study determined the prevalence of menopausal symptoms and not the severity of the symptoms.

All women were interviewed in Malay language. Faceto-face interview were done on all the women by trained 
health personnel who had undergone training, as this was important to make sure right answer were given, and explanations can be given if the women were in doubt or unclear about the questions asked. At the reception desk during registration, women who fulfill the criteria were invited to participate in this study. Explanations were given and written informed consent was taken from them.

\section{Statistical analysis}

The Statistical Package for the Social Sciences software Version 14.0 (SPSS, Chicago, IL) was used for univariate analyses. The $X^{2}$ test was applied to compare the frequencies of the symptoms among the different menopausal status. The level $\mathrm{P}<0.05$ was considered as the cut-off value for significance.

\section{Results}

Three hundred and fifty six women completed the study. The mean age of respondents in this study was $50.83 \pm 6.30(\mathrm{SD})$ years. The mean age at menopause was $51.28 \pm 2.28(\mathrm{SD})$ years with median of 50 years. Among these women, 82 (23.0\%) were premenopausal, 141 (39.6\%) perimenopausal and 133 (37.4\%) postmenopausal. The Iban, Chinese and Bidayuh were the majority ethnic group in this study. Christians made up 178 (50.0\%) of the participants, 256 (71.9\%) were married and $319(89.6 \%)$ had 11 or less years of schooling. Majority 164 (46.1\%) of the participants were housewives and most of the participants 169 (47.5\%) had a monthly household income below RM 1000 (US\$ 312). $(1 \mathrm{USD}=$ RM 3.2). (Table 1$)$

Table 2 shows the frequency of menopausal symptoms as assessed by the modified MRS according to most frequent complaints. The three most prevalent menopausal symptoms for all women $(\mathrm{n}=356)$ were: joint and muscular discomfort 285 (80.1\%), physical and mental exhaustion $239(67.1 \%)$ and sleeping problems 186 (52.2\%). This was followed by symptoms of hot flushes and sweating 148(41.6\%), irritability 135 (37.9\%), dryness of vagina 135 (37.9\%), anxiety 130 (36.5\%), depressive mood 116 (32.6\%), sexual problem 110 (30.9\%), bladder problems 49 (13.8\%) and heart discomfort/palpitation 65 (18.3\%).

Most of the somatic and psychological subscale symptoms occur in the perimenopausal group of women compared to pre- and postmenopausal women. However urogenital symptoms occurred most in postmenopausal women.(Table 3).

\section{Discussion}

The mean age at menopause in this study was $51.28 \pm$ 2.28 years. Although this is slightly higher than studies done in Peninsular Malaysia which reported mean age
Table 1 Socidemographic data of participants

\begin{tabular}{|c|c|}
\hline $\begin{array}{l}\text { Sociodemographic } \\
\text { Data }\end{array}$ & Total $(n=356)$ \\
\hline \multicolumn{2}{|l|}{ Age Group(years) } \\
\hline $40-44$ & 65 \\
\hline $45-49$ & 100 \\
\hline $50-54$ & 81 \\
\hline $55-59$ & 73 \\
\hline $60-65$ & 37 \\
\hline
\end{tabular}

$\begin{array}{lrr}\text { Ethnic Distribution } & & \\ \text { Iban } & 101 & 28.4 \\ \text { Chinese } & 80 & 22.4 \\ \text { Bidayuh } & 68 & 19.1 \\ \text { Malay } & 57 & 16.0 \\ \text { Melanau } & 22 & 6.2 \\ \text { Others(Lumbawang, } & 28 & 7.8\end{array}$

Kelabit, Kayan,

Penan, Punan)

Religion

Christian

Muslim

Buddhist

Others

Marital status

Married

Widow/divorcee

Single

Educational level

No formal

education

Primary level

50.0

21.1

22.6

22.6
6.3

Secondary level

Tertiary level

Occupation

Housewife

General worker

Semi-professional

Professional

$\begin{array}{rr}78 & 21.9 \\ 96 & 27.0 \\ 145 & 40.7 \\ 37 & 10.4 \\ & \\ 164 & 46.1 \\ 48 & 13.4 \\ 107 & 30.1 \\ 37 & 10.4\end{array}$

Household income (monthly)

Below RM 1000

RM 1001 - RM 2000

RM 2001 - RM 3000

RM 3001 - RM 4000

RM 4001 - RM 5000

Above RM 5000
27.0

40.7

10.4

46.1

13.4

30.1 $\begin{array}{r}47.5 \\ 19.4 \\ 21.9 \\ 6.7 \\ 3.4 \\ 1.1 \\ \hline\end{array}$ 
Table 2 Frequency of menopausal symptoms among Sarawakian women aged 40-65 years in Kuching, Sarawak assessed by modified MRS

\begin{tabular}{lrr}
\hline Menopausal symptoms & $\mathbf{n =} \mathbf{3 5 6}$ & $\mathbf{1 0 0 \%}$ \\
\hline 1. Joint and muscular discomfort & 285 & 80.1 \\
2. Physical and mental exhaustion & 239 & 67.1 \\
3. Sleeping problems & 186 & 52.2 \\
4. Hot flushes, sweating & 148 & 41.6 \\
5. Irritability & 135 & 37.9 \\
6. Dryness of vagina & 135 & 37.9 \\
7. Anxiety & 130 & 36.5 \\
8. Dpressive mood & 116 & 32.6 \\
9. Sexual problems & 110 & 30.9 \\
10. Heart discomfort & 65 & 18.3 \\
11. Bladder problems & 49 & 13.8 \\
\hline
\end{tabular}

of menopause between 49.4 to 51.1 years and from studies done in Thailand (48.7 years), Singapore (49.1 years) and other studies on Asian and Caucasian women, our findings still falls between the normal range of menopausal age $[3,9,10,13,17,18,20,21]$.

The assessment tool that we used in our study was based on Menopause Rating Scale (MRS) questionnaire. Although in menopausal symptoms studies few assessment tools were available, we used the Menopause Rating Scale (MRS) questionnaires, these questionnaires has been widely used in many epidemiological and clinical research when investigating the menopausal symptoms, These questionnaires has been validated and translated in many languages, although it is a self-administrated questionnaires, it's used were not only meant to assess the menopausal symptoms but also its severity, however, in our study, modification has to be done on the scaling of the original MRS because we noted that the respondents had difficulties in rating the scales, this could be explained by the fact that nearly half of the respondent studied never had formal education or only studied at primary level, and to minimize the reporting error, face to face interviewed were used instead of self administered by the respondents $[15,16]$.

In our study, the classical presentation of menopausal symptoms; hot flushes, sweating and night sweats were noted to be lower (41.6\%) when compared to findings from studies done on western women who were reported to be from $45 \%$ to $75 \%$. Similar result were also noted in two other studies done in Malaysia by Dhillon et al. (53.0\%) and Ismail (57.10\%), however, our finding of low menopausal classical symptoms were shared by studies done in other Asian countries [3,17,18,24-28].

In this study perimenopausal women were noted to experience more of vasomotor symptoms when compared to other menopausal group of women, and this was also statistical significant (Table 3), this can be explained by the fact that in these group of women, estrogen fluctuation during this phase occurs the most, hence they will experienced the most vasomotor symptoms. Our findings were correspond to studies conducted among Australia and other Caucasian women;

Table 3 Frequency of menopausal symptoms in the participants according to menopausal status among Sarawakian women aged 40-65 using the modified MRS

\begin{tabular}{|c|c|c|c|c|}
\hline Subscale (menopausal symptoms) & All $(n=356)$ & Premenopausal $(n=82)$ & Perimenopausal $(n=141)$ & Postmenopausal $(n=133)$ \\
\hline \multicolumn{5}{|l|}{ Somatic } \\
\hline 1. Hot flushes, sweating & $148(41.6) *$ & 29(35.4) & $91(64.5) *, \dagger$ & $28(21.1)$ \\
\hline 2. Heart discomfort & $65(18.3)$ & $3(3.7)$ & $40(28.4) *,+$ & $22(16.5)$ \\
\hline 3. Sleeping problems & $186(52.2)$ & $24(29.2)$ & $94(66.7) *,+$ & $68(51.1)$ \\
\hline 11. Joint and muscular discomfort & 285(80.1) & $36(43.9)$ & $129(91.4) *$ & $120(90.2) \neq$ \\
\hline \multicolumn{5}{|l|}{ Psychological } \\
\hline 4. Depressive mood & 116(32.6) & 13(15.9) & $67(47.5)$ & $36(27.1) \neq$ \\
\hline 5. Irritability & 135(37.9) & $29(35.4)$ & $77(54.6) *,+$ & $29(21.8) \dagger \neq$ \\
\hline 6. Anxiety & 130(36.5) & $29(35.4)$ & $78(55.4) *,+$ & 23(17.3) \\
\hline 7. Physical and mental exhaustion & 239(67.1) & $36(43.4)$ & $107(75.9) *$ & $96(72.2) \neq$ \\
\hline \multicolumn{5}{|l|}{ Urogenital } \\
\hline 8. Sexual problems & 110(30.9) & $17(20.7)$ & $58(41.2)+$ & $35(26.3) \neq$ \\
\hline 9. Bladder problems & 49(13.8) & $8(9.7)$ & $14(9.9)$ & $27(20.3) F_{1}^{* *}$ \\
\hline 10. Dryness of vagina & 135(37.9) & $16(19.5)$ & $58(41.2) *$ & $61(45.9) \neq_{1}^{* *}$ \\
\hline
\end{tabular}

* Significant difference $p<0.05$ compared to premenopausal

† Significant difference $p<0.05$ compared to postmenopausal.

\# Significant difference $p<0.05$ compared to premenopausal.

** Significant difference $p<0.05$ compared to perimenopausal 
where as high as $75 \%$ of perimenopausal women experienced bothersome vasomotor symptoms at some point of their transitional period [24-30].

From our study, joints and muscular discomfort; physical and mental exhaustions and sleeping problems (Table: 2) which is from the somatic and psychological subscales were experienced most by perimenopausal followed by postmenopausal women and these was also statistical significant differences when compared to premenopausal women. These findings were also noted to be corresponding to studies conducted Asian and Caucasian women $[3,7,10,13,18,19,27,28,31,32]$.

It is interesting to note that in our study, as much as $35 \%$ to $45 \%$ of premenopausal women also reported similar symptoms (joint and muscular discomfort, physical and mental exhaustion, anxiety, depressing mood, irritability), this could be explained since most of the somatic or psychological symptoms experienced by these middle age women are not exclusively as a result of changes due to menopause alone, it's could also resulted from other physical, psychological or health related problems which is related to aging in these group of women which can represent as menopausal like symptoms $[7,13,22]$.

In urogential subscale (sexual problems, bladder problems and vaginal dryness), from our study the frequency of these symptoms were experienced mainly by postmenopausal group of women and it was also significant statistically when compared to other menopausal status (Table 3) and similar finding were documented from other studies [3,18,25,27,32].

Natural menopause may strongly contribute to sexual changes experienced by these women, however its need to be emphasized that there are numerous factors which contribute to declining sexual activities in middle age women following menopause [18,33-36].

In our study it was noted that somatic and psychological symptoms were experienced mainly by perimenopausal women compared to the postmenopausal or premenopausal women. However, in the urogenital or sexual symptoms, the postmenopausal women were reported to suffer the most compared to the other two groups and similar findings were reported from other studies [2-4,13,18].

There are several limitations of this study. Although attempts were made to ensure that the study population was as representative as possible of the general population of the Kuching, Sarawak, nevertheless it has to be stated owing to the sampling technique used this might not be entirely possible. Another limitation was, as this was a cross sectional study, it does not exclude other confounding effects of the natural aging process that may influence experience of symptoms. Thirdly, this study used modified MRS questionnaire, translated to
Malay, so there is the question of accuracy in translation, although this was done by a group of health-workers and language experts. Although MRS is a self reporting questionnaire, in view of substantial number of women studied does not have formal education, in order to include these illiterate women, interviews were used instead. In collecting data, women are asked to provide some retrospective information, such as menopausal symptoms experienced in the preceding one month, last menstruation etc. Hence recall bias is unavoidable, especially for some elderly women. A final limitation of this study is lack of information on regularity of menstruation. Some subjects could have been misclassified into the incorrect menopause status group.

\section{Conclusion}

The study of middle- age women from Kuching, Sarawak between the ages of 40 and 65 years using modified Menopause Rating Scale (MRS), showed that the mean age of menopause was $51.28 \pm 2.28$ years.

The menopausal symptoms experienced by them were similar to other Asian women but the prevalence classical menopausal symptoms of hot flushes, sweating was lower compared to studies on Caucasian women.

The most common symptoms reported were from the somatic subscale; joint and muscular discomfort, physical and mental exhaustion and sleeping problems.

The perimenopausal women had the most significant somatic complaints compared to postmenopausal and premenopausal women, while postmenopausal women had the most significant urogenital symptoms compared to pre- and perimenopausal women.

\section{Acknowledgements}

We wish to thank Universiti Kebangsaan Malaysia for the research grant for this study. We also like to thank Sarawak Health Department, all the

government polyclinics around Kuching for their assistant in conducting this study.

We would also like to thank the original authors of the Menopause Rating Scale (MRS) for the use of this instrument during this study.

\section{Author details}

${ }^{1}$ Department of Family Medicine, Faculty of Medicine and Health Sciences Universiti Malaysia Sarawak, Kuching, Malaysia. ${ }^{2}$ Faculty of Resource Science and Technology, Universiti Malaysia Sarawak, Kuching, Malaysia. ${ }^{3}$ Department of Family Medicine, International Medical University, Kuala Lumpur, Malaysia.

Authors' contributions

SARSA participated in the design, coordinating and carried out the study and drafted the manuscript.

SRZ performed the statistical analysis and drafted the manuscript.

VLKM participated in the design and coordinating of the study.

All authors read and approved the final manuscript.

\section{Competing interests}

The authors declare that they have no competing interests.

Received: 29 July 2009

Accepted: 22 February 2010 Published: 22 February 2010 


\section{References}

1. Williams RE, Levine KB, Kalilani L, Lewis J, Clark RV: Menopause-specific questionnaire assessment in US population-based study shows negative impact on health-related quality of life. Maturitas 2009, 62(2):153-9.

2. Lu J, Liu J, Eden J: The experience of menopausal symptoms by Arabic women in Sydney. Climacteric 2007, 10:72-7.

3. Peeyananjarassri K, Cheewadhanaraks S, Hubbard M, Zoa Manga R, Manocha R, Eden J: Menopausal Symptoms in a hospital-based sample of women in southern Thailand. Climacteric 2006, 9:23-29.

4. Chedraui P, Blümel JE, Baron G, Belzares E, Bencosme A, Calle A, et al: Impaired quality of life among middle aged women: A multicentre Latin American study. Maturitas 2008, 61(4):323-9.

5. Lee I, Wang HH: Pattern and related factors of self-care behavior among perimemenopausal women. Public Health Q 2001, 28:151-60.

6. Shanafelt TD, Barton DL, Adjei AA, Loprinzi CL: Pathophysiology and treatment of hot flashes. Mayo Clin Proc 2002, 77:1207-1218.

7. Porter M, Penny GC, Russell D, Russell E: A population based survey of women's experience of the menopause. Br J Obstet Gynaecol 1996, 103:1025-8.

8. Oddens BJ, Boulet MJ, Lehert P, Visser AP: Has the climacteric been medicalised? A study on the use of medication for climacteric complaints in four countries. Maturitas 1992, 15(December (3)):171-81.

9. Pan HA, Wu MH, Hsu CC, Yao BL, Huang KE: The perception of menopause among women in Taiwan. Maturitas 2002, 25:41(4):269-74

10. Boulet MJ, Oddens BJ, Lehert P, Verme HM, Visser AP: Climacteric and menopause in seven South-east Asian countries. Maturitas 1994, 19(October (3)):157-76

11. Fuh Jl, Wang SJ, Lu SR, Juang KD, Chiu LM: The Kinmen women-health investigation (KIWI): a menopausal study of a population aged 40-54. Maturitas 2001, 39:117-24.

12. Jong LF, Shun JW, Shiang RL, Kai DJ, Lung MC: The Kinmen women-health investigation (KIWI): a menopausal study of a population aged 40-54. Maturitas 2001, 39:117-12.

13. Harvey C, Bee HT, Chia CA, Ee MC, Yap SC, Seang MS: The prevalence of menopausal symptoms in a community in Singapore. Maturitas 2002, 41:275-282.

14. Lori AB, Crystal MS, Kavita N: Is This Women Perimenopausal. JAMA 2003, 289(7).

15. Green JG: Construction a standard climacteric scale. Maturitas 1998, 29:19-24.

16. Heinemann LAJ, Potthoff $P$, Schneider HP: International version of the menopause rating scale (MRS). Health Qual Life Outcomes 2003 1:28.

17. Ismail NN: A study on menopause in Malaysia. Maturitas 1994, 19(3):205-209.

18. Dhillon HK, Singh HJ, Rashidah S, Abdul Manaf H, Nik Mohd Zaki NM: Prevalence of menopausal symptoms in women in Kelantan, Malaysia. Maturitas 2006, 54:213-221.

19. Dhillon HK, Singh HJ, Nor Aliza AG: Sexual function in menopausal women in Kelantan, Malaysia. Maturitas 2005, 52:256-263.

20. Hamid A, Tey N, Ramli N: A study on age of menopause and menopausal symptoms among Malaysian women. Malaysian Journal of Reproductive Health 1989, 7(1):1-9.

21. Ismael NN: A study on the menopause in Malaysia. Maturitas 1994, 19:205-209.

22. World Health Organisation (WHO): Scientific Group on Research on the Menopause in the 1990s. Research on the menopause: Report of a WHO scientific group. WHO technical report series Geneva: WHO866.

23. Soules MR, Sherman S, Parrot E: Executive summary: stages of reproductive aging workshop (STRAW). J Women's Health Gender-based 2001, 10(9).

24. Takeshi A: First Consensus Meeting on Menopause in the East Asian Region: Demography of the menopause and pattern of climacteric symptoms in the East Asian region. Geneva Foundation for Medical Education and Research 2003.

25. Dennerstein L, Duddley EC, Hopper JL, Guthrie JR, Burger HG: A perspective population-based study of menopausal symptoms. Obstet Gynecol 2000, 96:351-358.

26. McKinlay SM, Brambilla DJ, Posner JG: The normal menopause transition. Maturitas 1992, 14:103-115.
27. Loh FH, Khin LW, Saw SM, Jeannette JM, Ken Gu: The age of menopause and the menopause transition in a multiracial population: a nation-wide Singapore study. Maturitas 2005, 52:169-180.

28. Lam PM, Leung TN, Haines C, Chung TK: Climacteric symptoms and knowledge about hormone replacement therapy among Hong Kong Chinese women aged 40-60 years. Maturitas 2003, 30:45(2):99-107.

29. Guthrie JR, Dennerstein L, Taffe JR, Lehert P, Burger HG: The menopausal transition: a 9-year prospective population-based study. The Melbourne Women's Midlife Health Project. Climacteric 2004, 7:375-389.

30. Travers C, O'Neill SM, King R, Battistuta D, Khoo SK: Green Climacteric Scale: norm in an Australian population in relation to age and menopausal status. Climacteric 2005, 8:56-62.

31. Gold EB, Block G, Crawford S: Lifestyle and demographic factors in relation to vasomotor symptoms: baseline results from the Study of Women's Health Across the Nation. Am J Epidemiol 2004, 159:1189-1199.

32. Park YJ, Kim HS, Chang SO, Kang HC, Chun SH: Sexuality and related factors of postmenopausal Korean women. Taehan Kanko Hakhoe Chi 2003, 33(4):457-63.

33. Greendale GA, Lee NP, Arriola ER: The menopase. Lancet 1999, 353:571-580.

34. Rekers H, Drogendijk AC, Valkenburg HA, Riphagen F: The menopause, urinary incontinence and other symptoms of the genitor-urinary tract. Maturitas 1992, 15:101-111.

35. Milsom I, Ekelund P, Molander U, Arvidsson L, Areskoug B: The influence of age, parity, oral contraception, hysterectomy and menopause on the prevalence of urinary incontinence in women. Journal of Urology 1993 149:1459-1462.

36. Sherburn M, Guthrie JR, Dudley EC, O'Connell HE, Dennerstein L: Is incontinence associated with menopause?. Obstet Gynecol 2001, 98:628-633.

doi:10.1186/1447-056X-9-5

Cite this article as: Rahman et al: Assessment of menopausal symptoms using modified Menopause Rating Scale (MRS) among middle age women in Kuching, Sarawak, Malaysia. Asia Pacific Family Medicine 2010 9:5.

\section{Submit your next manuscript to BioMed Central and take full advantage of:}

- Convenient online submission

- Thorough peer review

- No space constraints or color figure charges

- Immediate publication on acceptance

- Inclusion in PubMed, CAS, Scopus and Google Scholar

- Research which is freely available for redistribution

Submit your manuscript at www biomedcentral com/submit
Biomed Central 